\title{
Formation des professeurs de langues en région frontalière
}

croyances et représentations des futurs professeurs d'allemand en Alsace et l'enseignement de la langue du voisin

Julia Putsche et Chloé Faucompré

\section{(2) OpenEdition \\ Journals}

Édition électronique

URL : http://journals.openedition.org/esp/817

DOI : $10.4000 /$ esp. 817

ISSN : 2532-0319

Éditeur

Centre d'Information sur l'Éducation Bilingue et Plurilingue

Édition imprimée

Date de publication : 1 juin 2016

Pagination : 47-60

ISSN : $1127-266 \mathrm{X}$

Référence électronique

Julia Putsche et Chloé Faucompré, "Formation des professeurs de langues en région frontalière », Éducation et sociétés plurilingues [En ligne], 40 | 2016, mis en ligne le 28 octobre 2016, consulté le 01 mai 2019. URL : http://journals.openedition.org/esp/817 ; DOI : 10.4000/esp.817 


\section{FORMATION DES PROFESSEURS DE LANGUES EN RÉGION FRONTALIÈRE: CROYANCES ET REPRÉSENTATIONS DES FUTURS PROFESSEURS D'ALLEMAND EN ALSACE ET L'ENSEIGNEMENT DE LA LANGUE DU VOISIN}

\section{Julia Putsche, Chloé Faucompré}

Tratteremo qui della situazione sociodidattica peculiare dei futuri insegnanti di lingua straniera nella regione frontaliera francotedesca d'Alsazia e del Baden-Württemberg. Alcuni visultati della ricerca sottolineano la necessità di applicare una didattica delle lingue "transfrontaliera". Esistono già alcum modelli teorici per questo tipo di approccio, ma manca una concreta proposta. Nel nostro progetto franco-tedesco di ricerca/insegnamento condotto congiuntamente a Strasburgo e a Friburgo abbiamo concepito un corso d'apprendimento delle lingue basato sulla ricerca per i futuri insegnanti della "ingua del vicino". L'articolo analizza il progetto e la sua realizzazione, oltre alle credenze degli insegnanti su questo tipo di pedagogia.

Parole chiave: sociodidattica; Alsazia; Baden-Württemberg; didattica transfrontaliera; futuri insegnanti; lingua del vicino; pedagogia

Der vorliegende beitrag beschäftigt sich mit der besonderen soziodidaktischen Situation zukïnftiger FremdsprachenlehrerInnen in der deutsch-französischen grenzregion Elsass-baden-Wïrttemberg. Forschungsarbeiten der letzten Fahre verlangen nach einer Implementierung einer ,grenzdidaktik" für den nachbarsprachlichen unterricht, doch neben theoretischen Modellen hierzu, lagen bislang keine konkreten umsetzungen dazu vor. In unserem deutsch-französischen Forschungs-und Lehrprojekt zwischen der université de Strasbourg und der Pädagogischen Hochschule Freiburg, haben wir ein Lehrmodul nach dem Prinzip des Forschenden Lernens fiur zukïnftige Lehrkräfte der Nachbarsprache erstellt. Der vorliegende beitrag analysiert das Projekt, seine umsetzung und die Einstellungen der Probanden zur Thematik.

Schüsselwörter: Soziodidaktik, Elsass, baden-Württemberg, Grenzdidaktik, Nachbarsprache

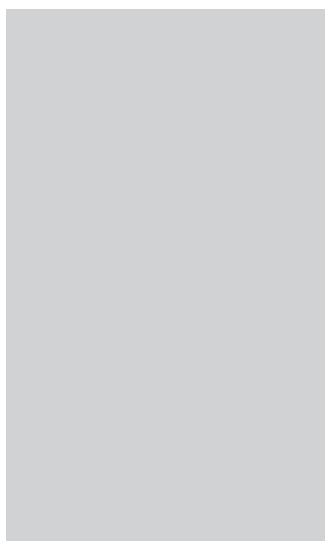

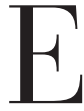

$\mathrm{n}$ région frontalière se croisent au minimum deux langues et deux cultures entrant en interaction l'une avec l'autre. Dans la région du Rhin supérieur, les travaux de référence de BothorelWitz (2008) ont montré à plusieurs reprises la situation sociolinguistique (notamment dans les entreprises du bassin alsacien du Rhin supérieur) de l'espace en question ainsi que l'omniprésence du contact entre les langues et cultures voisines. Les recherches de Huck, Bothorel-Witz et Geiger-Jaillet (2007) analysent la situation de l'enseignement de la langue du voisin des deux côtés du Rhin. Dans ces travaux scientifiques, l'accent est soit mis sur l'aspect historique, soit sur l'aspect analytique en examinant en détail la position des politiques linguistiques des régions en question (Alsace, Bade-Wurtemberg, Palatinat) ou bien en analysant les situations d'enseignement-apprentissage chez les apprenants de différents âges (voir aussi Putsche 2011). 
Formation des professeurs de langues en région frontalière.

J. Putsche, C. Faucompré

QU'EST-CE QU'UNE DIDACTIQUE TRANSFRONTALIÈRE?
La perspective des enseignants et leur vision de ce contexte particulier et de ses besoins spécifiques n'ont pourtant pas encore été traitées, alors que l'enseignement de la langue du voisin et l'ouverture au plurilinguisme prennent une dimension toute particulière en raison de la proximité géographique, permettant une réflexion et un travail approfondi sur la question de l'altérité. Nos expériences en tant qu'enseignantes et enseignantes-chercheures travaillant dans la formation des futurs enseignants de la langue du voisin dans la région en question, nous ont poussées à approfondir et à diriger notre recherche dans cette voie. C'est pourquoi nous nous sommes posé la question suivante: Comment sensibiliser les futurs professeurs de langues à l'enseignement en région frontalière?

Des travaux scientifiques antérieurs ont révélé que la proximité géographique avec le pays voisin n'était pas toujours synonyme de représentations positives chez les élèves par rapport à la langue-culture voisine (Raasch 2005, 2008; Putsche 2011). Tout au long de son parcours scolaire en tant qu'apprenant de la langue du voisin, mais aussi en tant qu'habitant d'une région frontalière, l'étudiant va se construire des représentations sociales mais aussi culturelles de cette langue, qui influeront par la suite sur son enseignement (Castelloti \& Moore 2002). Il convient par conséquent d'agir dès la formation initiale des futurs professeurs de langues pour appréhender la problématique à sa racine. Tout au long de cet article, nous analyserons la construction des représentations des futurs professeurs d'allemand (participants en Master 2) en formation à l'université de Strasbourg dans ce contexte frontalier, en nous focalisant sur la cognition enseignante (Borg 2006). Nous présenterons ensuite un modèle d'enseignement que nous avons élaboré et mis en place tout au long d'un semestre, afin de sensibiliser les apprenants aux caractéristiques de l'enseignement des langues en région frontalière. L'article s'appuie sur une expérimentation en cours dont la récolte des données est terminée mais dont l'analyse et l'interprétation des données ne le sont pas encore intégralement.

Afin de donner une explication brève mais précise du terme «didactique transfrontalière», il est important de prendre en considération les travaux d'Albert Raasch et notamment son modèle en cinq étapes décrivant ce à quoi peut correspondre une compétence transfrontalière. Raasch s'est beaucoup interrogé sur l'optimisation de l'enseignement de la langue du voisin dans les régions frontalières franco-allemandes. Il propose un modèle de «compétence transfrontalière» à acquérir au fil du temps, grâce au cours de langue dispensé en région frontalière. Pour ce faire, il souligne également l'importance d'avoir des enseignants de langue conscients de la relation entre langue et culture (Raasch 2002). Le modèle théorique de cette compétence frontalière 
Formation des professeurs de langues en région frontalière.

J. Putsche, C. FAucompré

\section{LA COGNITION ENSEIGNANTE}

commence par la transmission de compétences civilisationnelles (faits culturels, coutumes etc. de la région voisine). Dans un deuxième temps, les compétences civilisationnelles contrastives permettent aux apprenants de pouvoir comparer leur propre culture à celle d'en face. Suit la troisième étape, la compétence empathique, qui consiste en la capacité de pouvoir ressentir de l'empathie pour la culture voisine et, plus précisément, pour les citoyens voisins. La quatrième compétence est la compétence interculturelle, notion évidente et déjà bien définie dans le domaine de la didactique des langues. La cinquième étape est la compétence intra-culturelle, conception selon laquelle, l'apprenant devient capable de s'identifier à l'espace transfrontalier dans lequel il vit en tant que tel, c'est-à-dire l'espace géographique qui englobe ainsi les deux côtés de la frontière.

Notre dispositif d'enseignement universitaire s'est déroulé de la manière suivante: nous avons proposé une première phase d'enseignement lors de laquelle la présentation du concept, la lecture de textes scientifiques et le débat sur ces concepts ont mené à une articulation concrète et didactique de la notion pour que, dans un deuxième temps, les participants puissent mettre en œuvre les savoirs dans le but de créer des séances de cours de DaF (Deutsch als Fremdsprache (allemand langue étrangère) prenant en compte les éléments propres à cette didactique.

Simon Borg (2006) emploie le terme de cognition enseignante chez les enseignants de langues pour décrire toutes les facettes cognitives, ainsi que les différents facteurs externes qui influencent ce que pensent les enseignants. Borg résume le concept en expliquant que les recherches dans le domaine de la cognition enseignante, ne s'intéressent pas exclusivement à ce qui se passe pendant le cours de langue, mais plutôt à ce que l'enseignant lui-même décide, pense, ressent et vit.

"Teacher cognition research is concerned with understanding what teachers think, know and believe. Its primary concern, therefore, lies with the unobservable dimension of teaching teachers' mental lives.[...] Developments in cognitive psychology highlighted complex relationships between what people do and what they know and believe; educational researchers thus became more aware of the fact that in teaching too, teachers' mental lives played a role in their instructional choices. In other words, teachers were not robots who simply implemented, in an unthinking manner, curricula designed by others; rather, teachers exerted agency in the classroom - they made decisions, both before and while teaching and these decisions thus became a new focus for educational researchers. The questions being addressed now were not simply 'what do teachers do?' but also 'what do they think?', 'what decisions do they make?' and 'why?"' (Borg 2006: 1).

Dans notre étude, nous mettons l'accent sur les représentations de 
Formation des professeurs de langues en région frontalière.

J. Putsche, C. FAucompré

\section{MÉTHODOLOGIE}

\section{DÉROULEMENT \\ DU PROJET}

PRISE

D'INFORMATION futurs enseignants, c'est-à-dire que nous nous attardons sur la manière dont ils pensent l'enseignement de la langue-culture du voisin allemand.

La méthodologie de recherche, dont nous présentons un extrait dans cet article, s'articule autour d'une collaboration scientifique et pratique sur deux terrains universitaires et dans deux contextes régionaux et nationaux différents (1). Nous avons pour objectif de confronter les données de ces deux terrains dans une logique d'effet miroir, où les didactisations des participants strasbourgeois sont présentées aux participants fribourgeois et réciproquement. C'est-à-dire qu'il ne s'agit ni d'une comparaison, ni d'une juxtaposition des deux terrains, mais bien d'une volonté de comprendre deux phénomènes distincts dans un même espace européen, où la distinction s'articule notamment dans les programmes, dans la culture scolaire, au niveau du matériel d'enseignement, dans la formation des enseignants, etc. Dans ce type de dispositif de recherche, l'objectif ne consiste pas à faire une sorte de melting pot de deux contextes différents, mais si nous nous positionnons dans une perspective européenne avec comme objectif une identité commune en plus de l'identité nationale ou régionale, il est nécessaire, au sein d'une région frontalière, d'analyser la situation des deux côtés de la frontière.

Nous ne présenterons dans cet article qu'une partie des résultats récoltés sur le terrain alsacien (la récolte des données en Allemagne a commencé seulement après la finalisation de cet article).

L'objectif consiste en une approche de Forschendes Lernen (Huber 2009) ou, pour utiliser le terme anglais, research based learning. Le terme allemand décrit un dispositif d'enseignement universitaire dans le cadre duquel les participants n'apprennent pas seulement grâce à l'input scientifique de l'enseignant-chercheur, mais deviennent eux-mêmes «chercheurs» en proposant des solutions à un problème donné. Pour donner une traduction intelligible de ce terme, sans en changer la signification, nous proposons l'expression «apprendre par la recherche». Afin d'expliciter le dispositif, nous présentons ci-dessous les différentes étapes du projet.

La méthodologie de recherche élaborée pour notre projet se dessine en quatre étapes, réparties sur un semestre universitaire (12 semaines en raison de deux heures hebdomadaires; il s'agit du 2ème semestre de Master2). Nous présenterons les différentes étapes dans l'ordre chronologique.

Avant de commencer l'enseignement portant sur une possible didactique des langues transfrontalière, nous avons distribué un questionnaire semi-ouvert, contenant des questions destinées à nous renseigner sur 
Formation des professeurs de langues en région frontalière.

J. Putsche, C. Faucompré

INPUT SCIENTIFIQUE ET DIDACTIQUE

\section{DIDACTISATIONS}

\section{PRISE}

D'INFORMATION FINALE les représentations et attitudes des participants face à la région frontalière et à l'enseignement de la langue du voisin dans cette région. Il comprend des questions portant aussi bien sur les pratiques d'enseignement que sur le vécu (personnel et professionnel) des participants dans la région frontalière. La dernière question interroge les participants sur l'image concrète qu'ils se font d'une possible didactique des langues transfrontalière. Cette première étape permet de récolter des informations quant aux représentations spontanées et au vécu des participants. Elle constitue en quelque sorte une image instantanée in situ au début du projet.

Lors des six premières séances de cours, une phase d'input scientifique a été proposée aux participants, reprenant et expliquant les différents aspects de ce qu'est une didactique des langues transfrontalière, en travaillant principalement sur des textes scientifiques. En plus de cette transmission de savoir, une conférence sur une étude de cas (Faucompré 2014) menée du côté allemand a montré aux participants une possible mise en place de façon concrète. La conférencière a présenté une possible didactisation de différents contenus culturels et langagiers adapté au contexte géographique en question, dans le but de donner à la fois des pistes didactiques aux participants et de proposer un premier échange sur le sujet.

La troisième étape (étalée sur 4 semaines) consistait en la didactisation d'un contenu langagier et/ou culturel au choix dans une logique transfrontalière. Les participants avaient pour tâche de créer une séance de cours dans une logique transfrontalière pour le public de leur choix. Ils ont présenté leurs didactisations en plénum afin d'avoir les retours de la part de l'enseignante ainsi que des autres participants. Tous les supports de ces séances de cours, créées et imaginées par les participants, ont ensuite été partagées sur la plateforme d'apprentissage du groupe pour que chaque étudiant puisse profiter des créations de cours des autres.

À la fin du semestre, les participants ont de nouveau rempli un questionnaire semi-ouvert nous renseignant sur leur positionnement face à une possible didactique transfrontalière des langues, après y avoir été sensibilisés tout au long du semestre.

Le concept de l'enseignement et son articulation en savoirs, savoir-faire et savoir-être est pris dans une optique de Forschendes Lernen. Il s'agit dans un premier temps d'une simple transmission de savoirs par l'enseignant-chercheur qui doit donner la possibilité à l'étudiant de développer un savoir-faire, c'est-à-dire, la création de séances de cours dans 
Formation des professeurs de langues en région frontalière.

J. Putsche, C. Faucompré

\section{POSITIONNEMENT} DU CHERCHEUR

\section{COHORTE}

une logique transfrontalière. Le résultat - la proposition de solutionproduit à la situation-problème - se compose d'une didactisation d'un contenu langagier et/ou culturel, de la capacité à créer ce type de cours, ainsi que de choisir les supports adéquats pour le réaliser.

De plus, il s'agit également d'un savoir-être reprenant le concept de conscientisation qui est importante chez l'enseignant de langues en général et chez l'enseignant de langues en région frontalière en particulier (Raasch 2002: 14).

Comme nous l'avons déjà évoqué, le projet de recherche reprend des éléments d'une Recherche-Action sans en être une à proprement parler.

Enfin, la recherche-action en didactique des langues peut souvent être considérée comme un système ouvert et non fermé, c'est-à-dire comme un espace de création et non comme un simple champ d'intervention. Un système ouvert offre la possibilité d'apports complémentaires, voire d'hybridations. Le pari d'une telle conception repose sur la conviction que les praticiens peuvent travailler de manière autonome (Macaire 2007: 106).

La volonté de changement (recherche engagée) est du côté des chercheurs-enseignants auprès de la cohorte de participants. Ceci demande à la fois une réflexion antérieure et une conscientisation de la part du chercheur. L'objectif primaire dans une telle recherche consiste à préserver une certaine neutralité face aux données et une séparation précise entre l'enseignant (personne transmettant des savoirs et savoir-faire autour de la thématique choisie) et le chercheur (personne analysant les données, les productions didactiques et le projet intégral de façon neutre). Dans ce type de projet, c'est le côté «chercheur» qui doit analyser de façon critique le projet intégral (et non uniquement les données récoltées). Un autre aspect important à souligner est le fait que dans le dispositif, et dans chacun des deux contextes, une des deux chercheurs est toujours présente agissant en tant qu'enseignante auprès du groupe de participants. Le second chercheur peut de ce fait avoir un regard plus «neutre» sur les données de l'autre contexte et vice-versa, tout en partageant la totalité des données et tout en ayant conçu le projet ensemble.

La cohorte est composée de treize participants en Master 2 Allemand. Parmi eux, douze sont professeurs-stagiaires dans un établissement scolaire de l'enseignement secondaire en Alsace et étudient parallèlement à l'ESPE (Ecole supérieure du professorat et de l'éducation) ainsi qu'à l'Université. Une étudiante a validé son Master 1 mais n’a pas réussi le concours du CAPES, elle est par conséquent qualifiée d'étudiante en M2, «non-fonctionnaire-stagiaire». Le groupe se compose de neuf 
Formation des professeurs de langues en région frontalière.

J. Putsche, C. Faucompré

RÉSULTATS: VÉCU DE LA PROXIMITÉ FRONTALIÈRE

REPRÉSENTATIONS D'UNE POSSIBLE DIDACTIQUE TRANSFRONTALIÈRE DES LANGUES AU DÉBUT DU SEMESTRE femmes et trois hommes entre 22 et 47 ans. Les biographies langagières des participants varient beaucoup mais ne sont pas au cour de nos analyses dans cette contribution. Toutefois, il est important de souligner que seulement la moitié des participants a vécu à l'étranger (dans un pays germanophone) pendant un séjour Erasmus ou une année d'assistanat, la plupart du temps en Allemagne ou dans un autre pays germanophone.

La cohorte se compose de participants germanistes enseignant l'allemand dans la région frontalière du Rhin Supérieur. Tous les participants ont un contact régulier avec l'autre pays et profitent de la proximité géographique avec l'Allemagne. Pourtant les résultats n'en sont pas pour autant homogènes.

En réponse à la question «Allez-vous dans le pays voisin?», tous les participants ont répondu oui. C'est la fréquence du déplacement qui diffère (une personne va plusieurs fois par semaine en Allemagne, tandis que quatre y vont plusieurs fois par mois et six plusieurs fois par an).

Le vécu de la proximité avec l'Allemagne est donc hétérogène. Dans le groupe de participants, il y a tout aussi bien des participants binationaux ayant des liens familiaux avec le pays voisin, que des participants alsaciens qui vivent cette proximité de façon positive et depuis toujours, c'est-à-dire qu'ils ont l'habitude de cette proximité et la présence de l'Autre des deux côtés de la frontière. Le troisième profil qui se dégage, regroupe les participants venant d'autres régions de France, étant installés en Alsace, la plupart du temps à Strasbourg. Tous les participants disent profiter du côté pratique de la région frontalière, comme aller dans l'autre pays pour faire des achats par exemple. Beaucoup d'entre eux évoquent ensuite des visites chez la famille, les amis, les personnes rencontrées lors de programmes d'échange universitaire, etc.

Les loisirs (culturels, sportifs ou autres) sont également une raison de se rendre dans le pays voisin. En somme, les raisons pour lesquelles les participants se rendent le plus souvent en Allemagne sont de nature personnelle ou pratique, mais rarement de nature familiale et jamais pour des raisons professionnelles.

Les représentations autour d'une possible didactique transfrontalière des langues se reflètent dans plusieurs réponses du premier questionnaire. Nous avons choisi d'illustrer les résultats du corpus à l'aide de quatre réponses à la question suivante:

«Selon vous, est-ce que la région frontalière devrait être source d'inspiration pour l'élaboration des cours de langues en région frontalière? Si oui pourquoi? Si non pourquoi pas?»

En général, nous pouvons retenir qu'aucun des participants n’a choisi 
Formation des professeurs de langues en région frontalière.

J. Putsche, C. Faucompré de répondre par la négative. Deux participants expriment des craintes, contre dix questionnaires montrant des réponses positives.

A. «Oui = la frontière est dématérialisée; les différences culturelles (territoires, aménagements, langue sont immédiatement visibles); grande richesse culturelle $\rightarrow$ visites / excursions faciles; proximité de TROIS pays»

B. «Oui, c'est dans l'échange qu'est la richesse et la région frontalière a la chance de la proximité»

C. «Oui, car c'est malheureux de constater que les élèves en Alsace ne maitrisent pas mieux l'allemand que dans le reste de la France et qu'ils ne connaissent que Kehl en Allemagne (et rien d'autre)»

D. «Oui, nous pourrions tirer parti de deux façons d'enseigner différentes. En mettant en regard ces deux formes d'enseignement, il serait intéressant d'aboutir à une 3ème forme, spécifique à une région frontalière. Possibilités de «voir» ce qui se fait de l'autre côté, de s'y confronter».

En analysant les réponses des participants, nous constatons qu'il leur est difficile de cerner exactement le sens de la question posée en début de semestre car même la représentation du terme «didactique» reste encore floue pour eux. De plus, nous remarquons que leurs réponses prennent différentes directions. Toutefois les réponses choisies pour illustrer les tendances de la cohorte mettent en évidence ce qui fait l'objet de leurs représentations, tels que l'inexistence des frontières matérielles et la possibilité d'une ouverture, d'un va et vient entre les cultures ainsi que l'idée d'échange. Il est à chaque fois intéressant de voir que l'attente de l'enseignant par rapport au niveau linguistique dans la langue cible diffère selon la situation géographique. Dans l'extrait C, l'étudiant déplore le fait que le niveau d'allemand soit tout aussi «faible» que celui des élèves français de «l'intérieur» et que les élèves ne connaissent que Kehl comme ville allemande. De notre point de vue scientifique et didactique, il nous semble plutôt normal et souhaitable que les apprenants de l'espace géographique frontalier aient d'abord un contact avec les villes et villages frontaliers avant de connaître d'autres endroits par le biais de leurs voyages scolaires ou autres déplacements dans le pays voisin. Nous constatons ici un exemple qui illustre bien les propos de Raasch (2005) qui explique que pour certaines personnes «le vrai pays commence seulement derrière la région frontalière en question». Dans l'avis $\mathrm{C}$, nous pouvons également noter une certaine déception chez la future enseignante par rapport au statut qu'elle attribue à la ville frontalière de Kehl. Il est vrai que les aspects relatifs à la civilisation en cours de langues sont souvent abordés dans la logique de la kognitive Landeskunde (2). Il est normal que la jeune enseignante, après son parcours universitaire dans une autre ville française que Strasbourg et un séjour en tant qu'assistante de français en Allemagne, ait une vision plus large, plus complète et plus «encyclopédique» de 
Formation des professeurs de langues en région frontalière.

J. Putsche, C. Faucompré

REPRÉSENTATIONS D'UNE POSSIBLE DIDACTIQUE TRANSFRONTALIÈRE À LA FIN DU SEMESTRE
l'Allemagne et de sa géographie. Néanmoins, les villes frontalières représentent la réalité géographique de cette Allemagne du Rhin supérieur à laquelle les apprenants pourraient avoir un réel accès en se servant de la proximité géographique.

Les représentations à la fin du semestre ont été récoltées à l'aide d'un deuxième questionnaire semi-ouvert. Il était composé de deux questions seulement. Treize questionnaires ont été remplis, parmi lesquels dix questionnaires reflétant des représentations positives voire très positives face à une didactique transfrontalière, et deux questionnaires présentant des avis plutôt sceptiques, voire négatifs.

À la première question «Que pensez-vous désormais d'une didactique des langues adaptée à la région frontalière?», dix participants ont exprimé leurs attitudes positives et / ou leur enthousiasme face au concept. Pour illustrer nos propos relatifs à l'analyse du corpus, nous présentons les avis des mêmes participants que pour la première série de questionnaire.

A. «Intéressant pour plusieurs raisons = matériaux faciles à obtenir; proximité = facilité d'accès (excursions); reconnaissance de la place des apprenants = confrontation / implication dans leur quotidien / avenir (professionnelculturel)»

B. «Très intéressant, à utiliser ou à prendre en considération dans le futur -> permettra de faire des unités d'enseignement plus proche de la réalité»

C. «À mon avis, il n'y a pas assez d'exemples de didactisations. De plus, cette didactique est presque exclusivement adaptée au collège, aux jeunes élèves, pas à un niveau lycée. Mais c'est vrai qu'il y a des bons côtés très pratiques comme comparer Strasbourg et Kehl. Cela devient concret pour les élèves.»

D. «Didactique intéressante, novatrice à mon sens. S'appuyer sur les particularités d'une eurorégion peut rendre l'apprentissage concret et motiver davantage les apprenants. Difficile pour les enseignants qui ne connaissent pas la région et viennent à peine d'arriver».

Dans les extraits retenus ici, nous voyons bien que le terme «didactique» devient plus clair pour les participants. Ceci est certainement dû à l'introduction de la terminologie proposée dans le cours au début du semestre, ainsi qu'à la visibilité et à la concrétisation d'un concept abstrait en un objet concret (cf. «solution-produit»).

Les didactisations constituent une véritable concrétisation d'un concept (qui jusque-là ne proposait aucune solution concrète; le modèle de Raasch étant une conceptualisation sans mise en pratique avant notre projet de recherche-action) et permettent aux participants une réelle prise de conscience. L'étape d'élaboration, qui suit l'input scientifique, 
Formation des professeurs de langues en région frontalière.

J. Putsche, C. Faucompré

\section{DISCUSSION}

et la distanciation à posteriori grâce aux réflexions et analyses des didactisations par le groupe des participants, les transforment véritablement en chercheurs ( $c f$. forschendes Lernen), les rendant également acteurs de leurs propres décisions didactiques, tout en leur donnant l'occasion d'adopter un positionnement critique.

Cette prise de conscience est déclenchée par les acteurs eux-mêmes (Rivière \& Cadet 2011) et semble un outil nécessaire pour une visée de changement de paradigme pour un contexte bien spécifique. Les extraits présentés ici révèlent aussi parfois le fait que les enseignants n'aient pas encore saisi l'importance de prendre eux-mêmes les initiatives en tant que «spécialiste» (=enseignant de la langue du voisin). Ils ne semblent pas être totalement convaincus par le fait de devoir se former et s'informer par eux-mêmes, de se doter d'une certaine curiosité pour cette partie du pays que représente la région frontalière qui a peut-être moins d'attrait pour eux au premier abord («difficile pour les enseignants qui ne connaissent pas la région et viennent à peine d'arriver»). Les enseignants constatent dans tous les cas le côté pratique et pragmatique de cette didactique dite transfrontalière à la fois pour eux-mêmes («matériaux faciles à obtenir»), et pour les apprenants «plus concret et motivant pour les apprenants»). Cet aspect nous semblait secondaire avant le début du projet. Néanmoins, nous avons remarqué que le fait d'obtenir facilement du matériel didactique et pédagogique était un argument important pour les enseignants. Cette importance accordée au matériel peut également être interprétée négativement car une telle didactique demande plus d'investissement que la «simple utilisation» du manuel de langues à disposition dans leur établissement scolaire.

Nous avons choisi quatre profils de participants représentant les avis positifs et négatifs existant au sein du corpus. En regardant la première série de questionnaires, nous constatons que les quatre participants sont du même avis quant au fait que la région frontalière en tant que telle soit une source d'inspiration pour l'élaboration du cours de langues. Différentes raisons sont mises en avant, comme la proximité géographique et le fait qu'il n'y ait plus de frontière à proprement parler; la volonté d'aller voir au-delà de la frontière et le fait de renforcer le niveau en allemand des apprenants alsaciens (qui devraient, selon l'informateur, avoir un niveau linguistique en allemand bien supérieur que les élèves de n’importe où ailleurs en France). L'accent est soit mis sur la situation géographique, soit sur les traits caractéristiques de l'apprenant adolescent.

Dans la deuxième série de questionnaires, les participants s'expriment après avoir acquis des connaissances et des compétences (savoirs, savoir- faire et savoir- être) dans le domaine d'une possible didactique 
Formation des professeurs de langues en région frontalière.

J. Putsche, C. Faucompré

CONCLUSION ET PERSPECTIVES transfrontalière et après avoir proposé des séances de cours incluant des éléments caractéristiques de cette didactique.

Ils réalisent l'accès facilité au thème qu'ils pourront aborder et au matériel authentique dont ils pourraient se servir. Selon eux, la motivation des apprenants, ainsi que des enseignants, serait d'apprendre des choses «concrètes» et de faire un travail «utile».

L'objectif du cours proposé aux participants ne consistait pas uniquement en une transmission de savoirs et d'un maximum d'input. Toutefois, nous pouvons nous demander si l'apprentissage aurait été aussi efficace s'il y avait eu plus d'input de notre côté ou plus d'exemples de didactisations. Il s'agit, selon nous, de montrer des exemples mais surtout d'éviter la schématisation et le phénomène consistant à proposer une collection d'activités à adapter sans les modifier (comme avec un manuel de langues). Nous sommes étonnées de lire dans les réponses des participants que l'approche ne se prête pas à l'enseignement de l'allemand au lycée. Nous devons aussi souligner que la majorité des didactisations proposées par les participants ont été conçues pour un public de collégiens. Nous aurions dû davantage veiller à ce qu'il y ait des propositions ou une réflexion commune sur l'application de l'approche didactique pour le niveau lycée. Seulement deux participants avaient proposé des didactisations pour des classes de lycéens (ce déséquilibre est probablement du au fait que la majorité des participants sont en poste en collège). Quant au «savoir» de l'enseignant sur la région d'en face ou de son origine (venant d'ailleurs de France par exemple), nous nous positionnons clairement dans la perspective de Raasch (2002), qui souligne la nécessité majeure de former ou d'avoir à disposition des enseignants conscients du rôle qu'ils ont à jouer et qui font le lien entre la langue et la culture. Il ne s'agit pas d'avoir étudié l'histoire et la situation politique, sociale et économique de la région voisine en détail; il est à nos yeux un devoir de la part de l'enseignant de s'y intéresser, d'être curieux, de (se) poser des questions ainsi que de nourrir en permanence ses propres connaissances pour s’interroger de façon consciente sur ses représentations et leur éventuelle influence sur le cours de langues et les apprenants. L'enseignant doit être en quelque sorte un facilitateur, un passeur entre deux cultures pour rendre ce cheminement possible et pour ne pas faire de son cours en région frontalière un enseignement de langues artificiel, comparable par exemple à une situation de laboratoire de langues, ou excluant totalement la dimension frontalière.

Il nous semble important de souligner l'apport d'un tel dispositif d'enseignement universitaire qui se veut research-based pour les participants. À nos yeux, le «vrai moment d'apprentissage» dans ce type de cours est 
Formation des professeurs de langues en région frontalière.

J. Putsche, C. Faucompré beaucoup plus efficace que dans un cours de type magistral ou dirigé. L'étudiant est en effet progressivement rendu autonome et responsable de ses choix didactiques et pédagogiques, ce qui est particulièrement important dans cette phase de leur formation universitaire et pratique. La capacité à se poser des questions sur l'utilisabilité d'un matériel ou d'un manuel, au lieu de l'appliquer sans y réfléchir au préalable et sans vérifier si le contexte (géographique, social ou autre) s'y prête est, à nos yeux, une compétence indispensable chez un enseignant. Il s'agit pour l'enseignant-chercheur de provoquer une certaine prise de conscience chez l'étudiant, c'est pourquoi il est juste de parler de recherche engagée.

Perrenoud (2012) explique distinctement pourquoi cette conscientisation chez l'enseignant est importante en soulignant que la pratique réflexive dans l'enseignement est primordiale, et en proposant d'initier les futurs enseignants à celle-ci dès leur formation initiale.

[....] un formateur intervenant dans le domaine transversal, didactique ou technologique, peut organiser dans son champ thématique des moments d'analyse des pratiques plus ciblées, pour connecter les savoirs qu'il apporte aux pratiques des participants, en particulier en formation continue. De même, il n'est pas interdit de réfléchir aux passerelles possibles entre l'analyse des pratiques et la construction de savoirs, notamment au gré d'un travail par situations-problèmes (Perrenoud 2012: 116).

Nous ajoutons à cette conclusion que nous nous opposons formellement à la demande ou aux suggestions de créer un manuel de langues adapté à la région frontalière. Il deviendrait selon nous très rapidement un nouveau modèle figé parmi d'autres, et n'inciterait pas les enseignants à réfléchir par eux-mêmes ou à faire des choix individuels, mieux adaptés au contexte dans lequel ils enseignent et à la réalité dans laquelle vivent leurs apprenants. Concernant les perspectives de recherche, il est intéressant de récolter les données fribourgeoises, de les analyser et de les mettre en relief avec les données strasbourgeoises. Il est également nécessaire de partager ces données avec les participants français et allemands afin de leur montrer ce qui se fait de l'autre côté du Rhin, ainsi que de voir comment s'articulent les dissemblances entre les différents contextes au sein de l'espace géographique commun.

Cependant, rien ne nous garantit que les participants utiliseront plus tard l'approche didactique proposée dans leurs cours d'allemand. Les contraintes de temps, la pression du programme, si souvent citée, les directions institutionnelles, etc. pourraient bien évidemment être un frein à la mise en place d'un enseignement didactiquement transfrontalier. Néanmoins, nous constatons une sensibilisation, voire une prise de conscience réussie, car l'évolution des représentations face à une didac- 
Formation des professeurs de langues en région frontalière.

J. Putsche, C. Faucompré tique des langues transfrontalière est somme toute positive. Ceci montre la nécessité d'un tel module d'enseignement dans la formation des futurs enseignants.

\section{BIBLIOGRAPHIE}

BORG, Simon. 2006. Teacher cognition and language education: research and practice. London: Continuum International Publishing.

BOTHOREL-WITZ, Arlette. 2008. Le plurilinguisme en Alsace: les représentations sociales comme ressources ou outils de la description sociolinguistique. Les Cahiers de l'ACEDLE vol. 5 n 1: 41-63.

CASTELLOTTI V. \& MOORE D. 2002. Représentations sociales des langues et enseignements. Guide pour l'élaboration des politiques linguistiques éducatives en Europe-de la diversité linguistique à l'éducation plurilingue. Division des politiques linguistiques [en ligne], 27 (10). Consulté le 19 mai 2014.

http://www.coe.int/T/DG4/linguistic/Source/CastellottiMooreFR.p df.

FAUCOMPRÉ C. 2014. Vers la mise en place d'une didactique transfrontalière dans un Gymnasium en région frontalière. Mémoire de Master 2, Université de Strasbourg: Manuscrit.

HUBER L. 2009. Warum forschendes Lernen möglich und nötig ist, pp. 9-35 in Huber et al. (coord.), Forschendes Lernen im Studium. Bielefeld: Universitätsverlag Webler.

HUCK D., BOTHOREL-WITZ A. \& GEIGER-JAILLET A. 2007. L'Alsace et ses langues. Éléments de descriptions d'une situation sociolinguistique en région frontalière, pp. 13-10 in Abel A. et alii., Aspects of multilingualism in European Boarder Regions. Bozen: Eurac.

MACAIRE D. 2007. Didactique des langues et recherche-action, Les Cahiers de l'ACEDLE, Vol. 4: 93-120.

PELANDA-SIMON H. 2001. Landeskundlicher Ansatz, pp. 41-55 in Helbig, G., Götz L., Henrici G., Krumm H.-J. (Hg.), Deutsch als Fremdsprache: Ein internationales Handbuch. Berlin, New York: Walter de Gruyter.

PERRENOUD P. 2012. Développer la pratique réflexive dans le métier d'ensègnant, Paris: ESF.

PUTSCHE J. 2011. Spracheinstellungen von Grundschülerinnen und Grundschülern in einer Grenzregion. Qualitative Untersuchung in zwei paritätisch unterrichteten ersten Klassen mit Zielsprache Französisch, Bern: Peter Lang. RAASCH A. 2002. L'Europe, les frontières et les langues, Strasbourg, Conseil de l'Europe. 


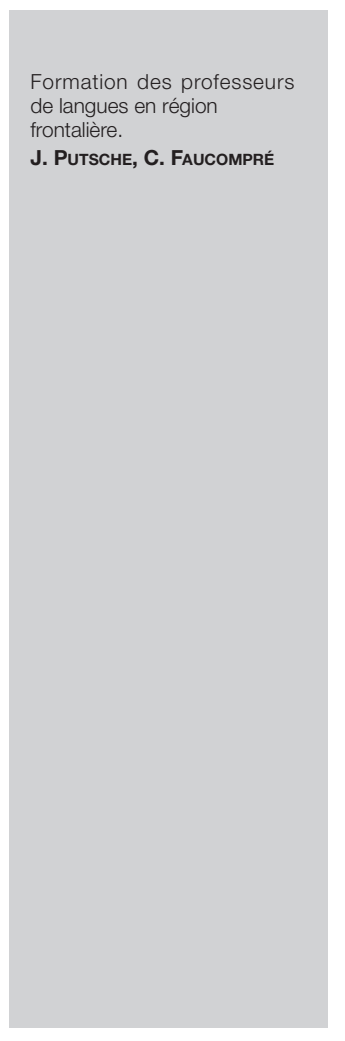

RAASCH A. 2005. Grenzkompetenz - ein Weg nach Europa. Wissenschaften von der Sprache und Kontextwissenschaften im Gespräch, pp. 119-129 in Denk R. (coord.), Nach Europa unterwegs. Grenzüberschreitende Modelle der Lehrerbildung im Zeichen von europäischer Identität, Kultur und Mehrsprachigkeit, Herbolzheim: Centaurus.

RAASCH A. 2008. Von Baden-Württemberg nach Europa und zurück Sprachenlernen und Sprachenlehren als sprachenpolitisches Handeln, pp. 21-40 in Windmüller F. (dir.), Synergies Pays germanophones $n^{\circ}$ 1. L'enseignement bi-plurilingue: Éducations, compétences, stratégies d'apprentissage. Berlin, Avinus.

RIVIERE V. \& CADET L. 2011. Mise en perspective des savoirs professionnels dans des discours de retour d'expérience. Étude contrastée de deux enseignantes de français langue étrangère. LIDIL 43: 41-55.

\section{NOTES}

(1) Nous proposons de façon identique (en s'adaptant à chaque contexte national ou régional), pendant un semestre, le même enseignement auprès de participants français et allemands se destinant à l'enseignement de la langue du voisin. Les participants de Strasbourg sont en Master 2 MEEF (allemand) et en même temps fonctionnaires-stagiaires de l'Éducation Nationale (ils enseignent l'allemand à hauteur de $9 \mathrm{~h} / \mathrm{semaine}$ dans un établissement d'enseignement secondaire de l'Académie de Strasbourg. Les participants de Fribourg-enBrisgau (Pädagogische Hochschule Freiburg) ont choisi dès le départ de leurs études supérieures la voie Lehramt (enseignement du français) et se trouvent au 7 ème et 8ème semestre (niveau master) de leur formation universitaire.

(2) Traduction: «civilisation cognitive»; dans son article, Hans Pelanda (2001: 47) présente les différentes approches de l'enseignement des aspects civilisationnels en cours de langues. L'approche cognitive vise la transmission d'un savoir «complet» qui reflète la «réalité» d'un pays et qui explique le fonctionnement politique et social, sintéressant aux institutions et structures d'organisation du pays dans une logique «encyclopédique». 